\title{
miR-19a-3p inhibition alleviates sepsis-induced lung injury via enhancing USP13 expression
}

\author{
Hangqi Ren', Wei Mu² and Qiaolian $\mathrm{Xu}^{3 \mathrm{~B}}$ \\ 'Department of Intensive Care Unit (ICU), The 942 Hospital of PLA, Yinchuan, Ningxia Hui Autonomous Region, 750001, China; ${ }^{2}$ Department of \\ Orthopaedics, The 942 Hospital of PLA, Yinchuan, Ningxia Hui Autonomous Region, 750001, China; ${ }^{3}$ Department of Intensive Care Unit (ICU), \\ Nanjing First Hospital-Nanjing Medical University, Nanjing, Jiangsu Province, 210009, China
}

Sepsis is a systemic inflammatory response syndrome caused by various pathogenic microorganisms or toxins. Lung damage is one of the causes of death in patients with sepsis. This study aimed to investigate the role of miR-19a-3p and its regulation mechanism in sepsisinduced lung injury. MH-S cells were treated with lipopolysaccharide (LPS) to establish sepsis-induced lung injury cell model. C57BL/6 mice were injected with miR$19 a-3 p$ antagomiR and LPS to construct animal model. LPS-treated and control cells were transfected with miR19a-3p mimic, miR-19a-3p inhibitor or USP13 expression vector. The expression levels of miR-19a-3p and USP13 were examined by quantitative real-time polymerase chain reaction (qRT-PCR) and Western blotting. The concentration of inflammatory cytokines was measured with enzyme-linked immunosorbent assay (ELISA). The relationship of miR-19a-3p and USP13 was validated using dual-luciferase reporter assay. The lung damage was assessed with hematoxylin-eosin staining (HE). The results showed that LPS treatment increased the concentration of TNF- $\alpha$, IL- 6 and IL-1 $\beta$ in MH-S cells. In LPS treated MH-S cells, the level of miR-19a-3p gradually increased over time. Both miR-19a-3p knockdown and USP13 overexpression in $\mathrm{MH}-\mathrm{S}$ cells inhibited the LPS-induced production of TNF- $a$, IL- 6 and IL-1 $\beta$. Moreover, miR-19a-3p negatively regulated the expression of USP13 in MH-S cells. Furthermore, miR-19a-3p inhibitor suppressed lung damage in sepsis model mice. In conclusion, miR-19a-3p knockdown could alleviate sepsis-induced lung injury through enhancing USP13 expression.

Keywords: miR-19a-3p, LPS; USP13, sepsis, lung injury

Received: 27 September, 2020; revised: 23 November, 2020; accepted: 24 November, 2020 available on-line: 10 May, 2021

$\square$ e-mail: xuqiaolian0918@163.com

Abbreviations: ANOVA, analysis of variance; ELISA, Enzyme-linked immunosorbent assay; EMT, epithelial-mesenchymal transformation ICU, Intensive Care Unit; LPS, lipopolysaccharide; MODS, multiple organ dysfunction syndrome; NC, nitrocellulose; qRT-PCR quantitative real-time polymerase chain reaction: $\mathrm{RPMI}$, Roswell Park Memorial Institute; SDS-PAGE, sodium dodecyl sulfate-polyacrylamide gel electrophoresis; SIRS, systemic inflammatory response syndrome; TBST, Tris-Buffered Saline Tween; USP13, ubiquitin-specific protease 13; 3'UTR, 3'-untranslated region

\section{INTRODUCTION}

Sepsis, a systemic inflammatory response syndrome (SIRS) in response to infectious process caused by various pathogenic microorganisms or released toxins, is a common complication of severe trauma and infections, and the main cause of death in the Intensive Care Unit
(ICU) (Dombrovskiy et al., 2007; Juroš et al., 2019). Sepsis can cause multiple organ dysfunction syndrome (MODS) (Mineji, 2018). The lungs are the most vulnerable organ in the course of MODS (Balthesen et al., 1993). At present, acute lung injury is one of the main causes of death in patients with sepsis (Stapleton \& Steinberg, 2007). Because of the complicated pathogenesis of sepsis, it is difficult to treat the patients with sepsis-induced tissue damage. At the same time, rapid and effective means of detection and treatment for lung injury caused by sepsis are still lacking (Opal, 2014). Therefore, it is necessary to elucidate the molecular pathways in sepsis for better diagnostics and treatment.

MicroRNAs (miRNAs) are small single-stranded RNA molecules with a length of 18-25 nucleotides, which are common in plants and animals (Sotiropoulou et al., 2009). MiRNAs can degrade or inhibit the translation of target mRNA by complementary pairing with the 3'-untranslated region (3'UTR) of target mRNA, thus negatively regulating protein expression (Sethi et al., 2013). A growing number of studies have found that miRNAs play an important regulatory role in sepsis. For example, the expression of miR-146a and miR-223 in serum of patients with sepsis is decreased, which has a high predictive value for sepsis (Wang et al., 2010). It has also been found that miR-494 promotes acute lung injury induced by sepsis (Ling et al., 2018). In addition, miR$19 a-3 p$ was found to be up-regulated in sepsis (Chen et al., 2019). However, the specific effect of miR-19a-3p in sepsis induced lung injury is unclear.

The ubiquitin-specific protease 13 (USP13) belongs to the family of ubiquitin-specific proteases encoded by the genes located in the region of $3 \mathrm{q} 26.2-\mathrm{q} 26.3$ on the long arm of chromosome 3 in human (Zhang et al., 2013). Some studies have found that USP13 can participate in lung damage induced by LPS and has a certain anti-inflammatory effect (Li et al., 2019).

Therefore, this study aims to investigate the role of miR-19a-3p and its regulation mechanism in sepsis-induced lung injury using the LPS-induced cell model in vitro and mouse model in vivo.

\section{MATERIALS AND METHODS}

\section{Cell treatment and transfection}

Murine alveolar MH-S macrophages (Mingzhoubio, Ningbo, China) were incubated in 90\% Roswell Park Memorial Institute (RPMI)-1640 medium (Thermo Fisher Scientific, Waltham, USA) and 10\% fetal bovine se- 
rum (FBS, Thermo Fisher Scientific) at $37^{\circ} \mathrm{C}$ with a cell density of $70-80 \%$.

$\mathrm{MH}-\mathrm{S}$ cell were treated with lipopolysaccharide (LPS, $1 \mu \mathrm{g} / \mathrm{mL}$, Solarbio, Beijing, China) for 6,12 , or $24 \mathrm{~h}$.

MiR-19a-3p inhibitor, negative control (NC) inhibitor, miR-19a-3p mimic, NC mimic, vector or USP13 expression vector were transfected into control or LPS-treated MH-S cells using Lipofectamine 3000 (Thermo Fisher Scientific).

\section{Mouse model}

C57BL/6 mice obtained from Experimental Animal Center of Southern Medical University (Guangzhou, China) at 6-8 weeks with a weight of 21-25 g were randomly divided into 3 groups (12 mice each): the sham group, the LPS + NC antagomiR group and the LPS + miR-19a-3p antagomiR group. The mice in latter two groups were injected with $25 \mathrm{nM}$ of NC antagomiR or miR-19a-3p antagomiR designed by GenePharma (Shanghai, China) through tail vein once a day for 3 consecutive days (Liu et al., 2018). After $24 \mathrm{~h}$ of the last injection, $1 \mathrm{mg} / \mathrm{kg}$ of LPS was injected into the trachea of the mice to carry out the follow-up experiments ( $\mathrm{Li}$ et al., 2019). Meanwhile, the mice in the sham group were injected with the equal amount of normal saline. Then lung tissues and alveolar lavage fluid were obtained from the mice. The sequence of miR-19a-3p antagomiR was 5'-TCAGT'T'T'TGCATAGAT'T'TGCACA-3', and the sequence of NC antagomiR was 5'-AAGGCAAGCUGACCCUGAAGUU-3'. All animal experiences were approved by the Ethics Committee of Nanjing First Hospital-Nanjing Medical University.

\section{Quantitative real-time polymerase chain reaction (qRT- PCR)}

The total RNA was extracted from alveolar lavage fluid and MH-S cells using TRIzolTM Reagent (Thermo Fisher Scientific) and reverse transcribed to cDNA using mRNA Selective PCR Kit (TaKaRa, Dalian, China). SYBR ${ }^{\mathrm{TM}}$ Green PCR MasterMix (Solarbio, Beijing, China) was used to detect the level of miR-19a-3p and USP13. The PCR primers of were purchased from Sangon Biotech Co., (Shanghai, China). The primers were: miR-19a-3p, Forward: 5'-AATAACTAGTAGGAAGCACTGT'TGGAGCTACTG-3'; Reverse: 5'-ATAAGCGGCCGCAGTCACCAAAATGTATTATAAGGG-3'; USP13, Forward: 5'-CCTGAATACT'TGGTAGTGCAGATAAAGAA-3'; Reverse: 5'-GCGCATGTT'TAAGGCCT'T'TGT-3'. The expression levels were normalized to $\mathrm{U} 6$ and calculated with the $2^{-\Delta \Delta \mathrm{Ct}}$ method (Livak \& Schmittgen, 2001).

\section{Enzyme-linked immunosorbent assay (ELISA)}

Supernatants from MH-S cell cultures were collected to detect tumor necrosis factor- $\alpha$ (TNF- $\alpha$ ), interleukin- 6 (IL-6) and IL-1 $\beta$ levels using ELISA Kit (Thermo Fisher Scientific). The absorbance was measured at $450 \mathrm{~nm}$ using a Microplate Reader (Bio-Rad, Hercules, USA).

\section{Dual-luciferase reporter assay}

$\mathrm{MH}-\mathrm{S}$ cells were co-transfected with miR-19a-3p mimic or NC mimic and pGL3-USP13-wild type (WT) or pGL3-USP13-mutant (MUT). Then, the luciferase activity was measured and analyzed according to DualLuciferase $^{\circledR}$ Reporter Assay System Protocol (Promega, USA).

\section{Western blotting}

Total protein was extracted from LPS-treated or control MH-S cells. Protein concentration was measured using Bradford method. The protein samples were preheated at $100^{\circ} \mathrm{C}$ for $5 \mathrm{~min}$, separated by $10 \%$ sodium dodecyl sulfate-polyacrylamide gel electrophoresis (SDSPAGE), and transferred into nitrocellulose (NC) membranes. The NC membranes were incubated with 5\% milk - TBST for $2 \mathrm{~h}$ at $37^{\circ} \mathrm{C}$, then co-incubated with USP13 antibody (ab109264, 1:1000, Abcam, Cambridge, USA) or $\beta$-actin antibody (ab213262, 1:1000, Abcam) for $16 \mathrm{~h}$ at $4^{\circ} \mathrm{C}$. The membranes were next washed four times with Tris-Buffered Saline Tween (TBST, Thermo Fisher Scientific) and incubated with the anti-rabbit IgG antibody (ab190492, $1 \mathrm{mg} / \mathrm{mL}, 1: 2000$, Abcam) conjugated with horseradish peroxidase for another $1 \mathrm{~h}$. The membranes were incubated in ECL and the exposed protein bands were observed and recorded using imaging system (Bio-Rad, USA). The bands were analyzed using Image J software (NIH Image, USA).

\section{Hematoxylin-eosin staining (HE)}

The mice were anesthetized and their lungs were removed by thoracotomy. The lung tissue was fixed with 4\% paraformaldehyde (PFA, Thermo Fisher Scientific) and sliced into 2-mm sections with a scalpel. The sections were dehydrated with ethanol gradient and dipped in paraffin for wax immersion. The hardened sections were cut into 5-8 $\mu \mathrm{m}$ slices using a slicer. The slices were scalded with hot water, and then dewaxed with xylene. After dewaxing and washing, the slices were stained in hematoxylin for $20 \mathrm{~min}$, fractionated in ethanol containing $1 \%$ hydrochloric acid for $3 \mathrm{~s}$, and reversed blue with $0.25 \%$ ammonia for $15 \mathrm{~s}$. The sections were rinsed with running water for $1 \mathrm{~h}$, dehydrated with $70 \%$ and $90 \%$ ethanol for $10 \mathrm{~min}$ each, and then stained with eosin for 2-3 min. These stained sections were dehydrated with ethanol, made transparent by xylene, and finally sealed with gums (all from Thermo Fisher Scientific). The stained lung tissue sections were analyzed using an optical microscope and photographed to record the results.

\section{Statistical analysis}

All data were analyzed with SPSS 21.0 software (SPSS Inc., USA). The $t$-test and analysis of variance (ANO$\mathrm{VA})$ were used to analyze the data. The results were presented as mean \pm standard deviation (S.D.). $p<0.05$ was considered statistically significant.

A

B
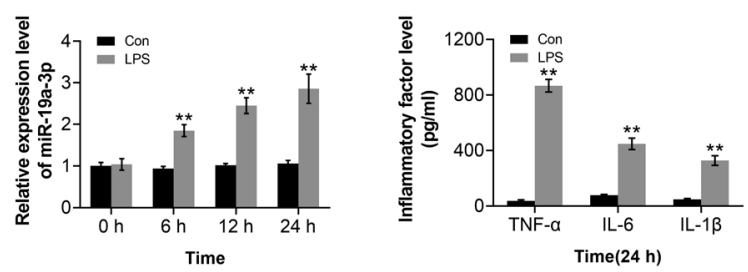

Figure 1. miR-19a-3p expression level was increased in LPS-induced $\mathrm{MH}-\mathrm{S}$ cells.

(A) The expression level of miR-19a-3p was measured by qRT-PCR in $\mathrm{MH}-\mathrm{S}$ cells treated with LPS for 6,12 and $24 \mathrm{~h}$ and in the control untreated cells (B) The levels of TNF-a, IL- 6 and IL-1 $\beta$ were measured with ELISA in MH-S cells treated with LPS for $24 \mathrm{~h}$. ${ }^{* *} p<0.01$. 
A

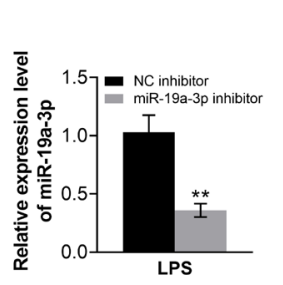

B

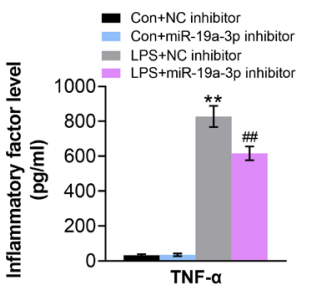

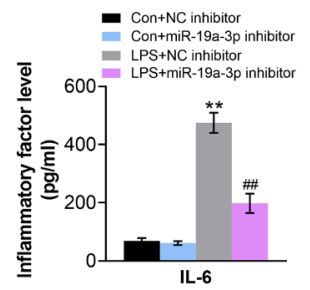

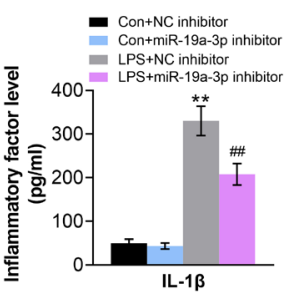

Figure 2. Knockdown of miR-19a-3p inhibited the production of inflammatory factors in LPS-induced MH-S cells.

MiR-19a-3p inhibitor or its negative control were transfected into control or LPS-treated MH-S cells. (A) The transfection efficiency of miR-19a-3p inhibitor was measured with qRT-PCR in LPS-treated MH-S cells. (B) The levels of TNF-a, IL-6 and IL-1 $\beta$ were measured with ELISA. ${ }^{* *} p<0.01,{ }^{*}$ describes the comparison of LPS $+\mathrm{NC}$ inhibitor and control $+\mathrm{NC}$ inhibitor groups. \#\# $p<0.01$, \#describes the comparison between LPS + miR-19a-3p inhibitor and LPS + NC inhibitor groups.

\section{RESULTS}

\section{miR-19a-3p was up-regulated in LPS-induced MH-S cells}

LPS was added to MH-S cell cultures to mimic sepsis-induced damage in vitro, and then the expression of miR-19a-3p was measured in LPS-stimulated MH-S cells. As shown in Fig. 1A, miR-19a-3p mRNA level gradually increased over time of LPS treatment $(p<0.01)$. Moreover, the concentration of secreted inflammatory cytokines (TNF- $\alpha$, IL- 6 and IL-1 $\beta$ ) was significantly higher in MH-S cells treated with LPS for $24 \mathrm{~h}$ compared to control $(p<0.01)$. Of these inflammatory factors, TNF- $\alpha$ increased the most (Fig. 1B). Thus, a sepsis-induced cell injury model was successfully established and in this model, LPS promoted high expression of miR-19a-3p.

\section{Knockdown of miR-19a-3p suppressed the production of inflammatory factors in LPS-induced MH-S cells}

To investigate the specific effects of miR-19a-3p inhibitor on intracellular inflammatory factors, miR-19a$3 \mathrm{p}$ inhibitor or its negative control were transfected into control or LPS-treated MH-S cells. As shown in Fig. 2A, miR-19a-3p relative expression level in LPSinduced cells was decreased after miR-19a-3p inhibitor transfection $(p<0.01)$. The concentration of TNF- $\alpha$, IL-6 and IL-1 $\beta$ levels measured with ELISA were not changed in control MH-S cells upon miR-19a-3p inhibitor transfection but the levels of TNF- $\alpha$, IL- 6 and IL-1 $\beta$ levels in LPS-induced cells were reduced $(p<0.01$, Fig. $2 \mathrm{~B})$. These results suggest that miR-19a-3p knockdown could inhibit the production of inflammatory factors in LPS-treated cells.

\section{A}

$\begin{array}{ll}\begin{array}{l}\text { Position 4256-4262 } \\ \text { USP13 3' UTR WT }\end{array} & 5^{\prime} \text {...UUGGUAUUUAUUUAUUUUGCACU...3' } \\ \text { mmu-miR-19a-3p } & 3^{\prime} \quad \text { AGUCAAAACGUAUCUAAACGUGU } 5^{\prime} \\ \text { USP13 3' UTR MUT } & 5^{\prime} \text {...UUGGUAUUUAUUUAUAAACGUGU...3' }\end{array}$

B

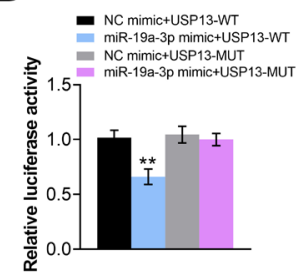

C

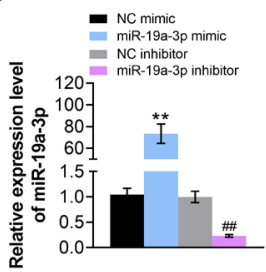

E

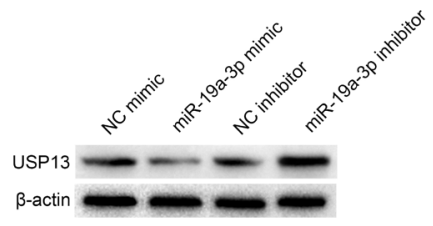

D
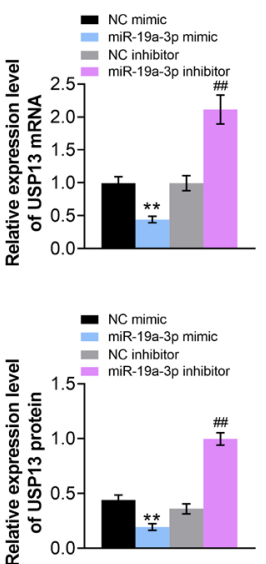

Figure 3. USP13 was a target of miR-19a-3p.

(A) The target site of miR-19a-3p in USP13 as predicted with TargetScan website. The panel shows the wild type and mutated sequence of USP13 3'UTR that were used in luciferase assays. (B) The targeting relationship between miR-19a-3p and USP13 was assessed by dual-luciferase reporter assay. (C) The transfection efficiency of miR-19a-3p inhibitor and miR-19a-3p mimic was measured with qRT-PCR (D) The USP13 mRNA level in MH-S cells transfected with miR-19a-3p inhibitor or miR-19a-3p mimic, as detected with qRT-PCR. (E) The USP13 protein level in transfected MH-S cells detected with Western blotting. ${ }^{* *} p<0.01,{ }^{*}$ describes the comparison of miR-19a-3p mimic group and NC mimic group. \# $p<0.01$, "describes the comparison of miR-19a-3p inhibitor group and NC inhibitor group. 

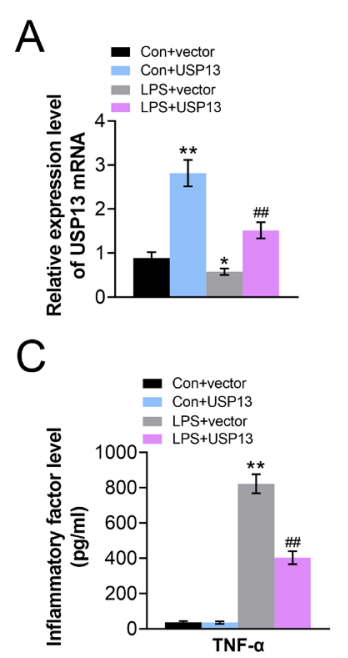

B
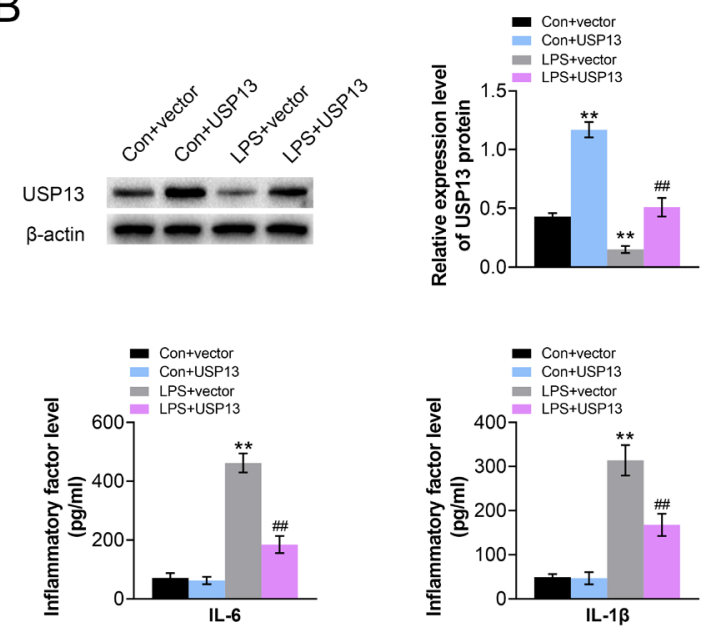

Figure 4. Overexpression of USP13 inhibited the production of inflammatory factors in LPS-induced MH-S cells.

USP13 expression vector was transfected into control and LPS-treated MH-S cells. (A) The mRNA level of USP13 in LPS-treated MH-S cells measured with qRT-PCR. (B) The USP13 protein levels detected with Western blotting. (C) The levels of TNF-a, IL-6 and IL-1 $\beta$ measured with ELISA. ${ }^{* *} p<0.01,{ }^{*}$ denotes the comparison of LPS + vector group and control + vector group. \#\# $<0.01$, \#denotes the comparison of LPS + USP13 group and LPS + vector group.

\section{USP13 was a target of miR-19a-3p in MH-S cells}

The target genes of $\mathrm{miR}-19 \mathrm{a}-3 \mathrm{p}$ were predicted at the TargetScan website (www.targetscan.org). One of these genes was USP13, in which several miR-19a-3p target sites were present (Fig. 3A). To experimentally verify if USP13 was a miR-19a-3p target, we applied luciferase assay, in which the cells were co-transfected with either miR-19a-3p mimic, or its negative control mimic, together with luciferase reporter gene construct fused to USP13 (Fig. 3B). In the cells co-transfected with miR19a-3p mimic and USP13-WT, the luciferase activity was lower compared to the cells co-transfected with negative control mimic and USP13-WT $(p<0.01)$. However, when a mutated sequence of USP13 was used in the luciferase reporter, there was no significant difference in luciferase activity between the cells co-transfected with the miR-19a-3p mimic and USP13-MUT and the cells co-transfected with the negative control mimic and USP13-MUT. The above results illustrated that USP13 was a target of miR-19a-3p. To observe a possible correlation between miR-19a-3p and USP13 expression in MH-S cells, miR-19a-3p mimic and miR-19a-3p inhibitor were transfected into MH-S cells (Fig. 3C), followed by qRT-PCR to assess miR-19a-3p and USP13 expression levels and Western blotting to detect USP13 protein levels in the transfected cells (Fig. 3D and 3E). miR-19a$3 p$ mimic decreased the expression level of USP13 both on mRNA and protein level, while miR-19a-3p suppressor had the opposite effect and significantly up-regulated USP13 expression $(p<0.01$, both for qRT-PCR and WB band intensities measurements). In summary, our data showed that miR-19a-3p could negatively regulate USP13 expression..

\section{Overexpression of USP13 inhibited the production of inflammatory factors in LPS-induced MH-S cells}

To investigate the influence of USP13 on inflammatory factors in sepsis, USP13 expression vector or negative control vector was transfected into LPS-treated MH-S cells. As shown in Fig. 4A and 4B, USP13 transfection of the MH-S cells resulted in an increase in USP13 mRNA and protein levels $\mathrm{t}(\phi<0.01)$. However, the overexpression of USP13 in MH-S cells stimulated by LPS was lower than that in control cells, indicating that LPS inhibited the expression of USP13. Interestingly, USP13 overexpression in control MH-S cells had no influence on TNF- $\alpha$, IL-6 and IL-1 $\beta$ concentration. In the LPS-treated MH-S cells, however, USP13 overexpression significantly reduced the otherwise elevated TNF- $\alpha$, IL- 6 and IL-1 $\beta$ expression levels $(p<0.01)$. Therefore, we conclude that high expression of USP13 could inhibit the secretion of inflammatory factors in the injury cell model.

\section{Knockdown of miR-19a-3p inhibited lung damage in septic mice}

To investigate the regulatory effects of miR-19a-3p on sepsis in vivo, the LPS-induced sepsis mouse model was established. As shown in Fig. 5A, LPS promoted the expression of miR-19a-3p, while reducing the mRNA level of USP13 in septic mice $(p<0.01)$. The above results were reversed with miR-19a-3p suppression $(p<0.01)$. Also the LPS-induced increase in TNF- $\alpha$, IL- 6 and IL$1 \beta$ levels was alleviated upon miR-19a-3p suppression, as shown with ELISA $(p<0.01$, Fig. $5 \mathrm{~B})$. On the tissue level, we observed that mice in the sham group had clear alveolar structures, their alveolar wall was thin, and there was no exudate or bleeding in the alveolar cavity (Fig. 5C), Mice in the LPS group experienced significant damage to the alveolar structures, the alveolar space was enlarged, and there was exudates and hemorrhage in the alveolar interstitial (Fig. 5C). Strikingly, he LPS-induced damage of alveolar structures and hemorrhage were relieved after inhibiting the expression of miR-19a-3p in LPS-treated mice. With these findings we conclude that miR-19a-3p could promote LPS-induced lung damage in the mouse model.

\section{DISCUSSION}

Sepsis is a life-threatening condition that leads to uncontrolled inflammation (Dombrovskiy et al., 2007). It has been found that miRNAs can regulate the imbalanced inflammatory response by down-regulating the inflammatory factors, their receptors and related signaling 
A
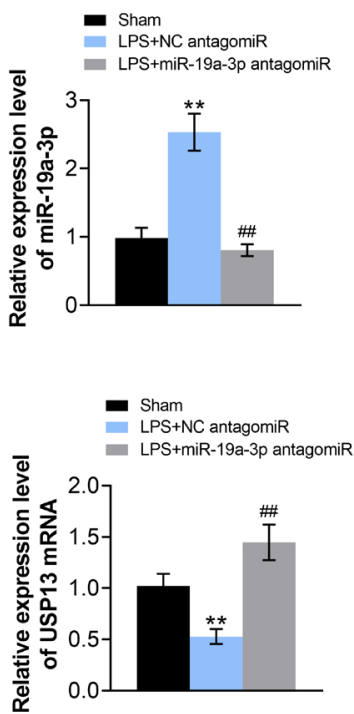

B

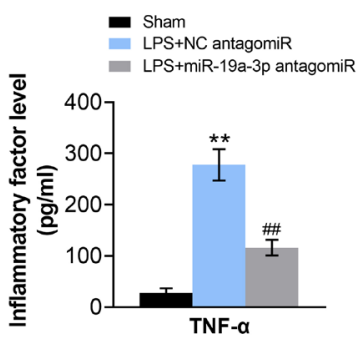

C

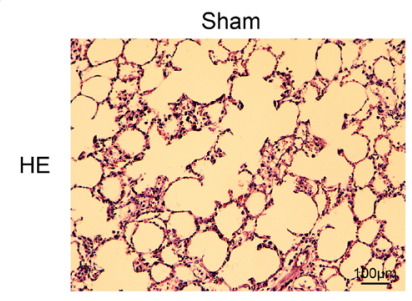

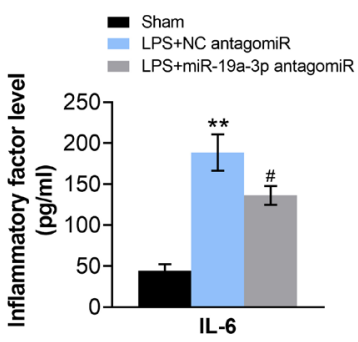
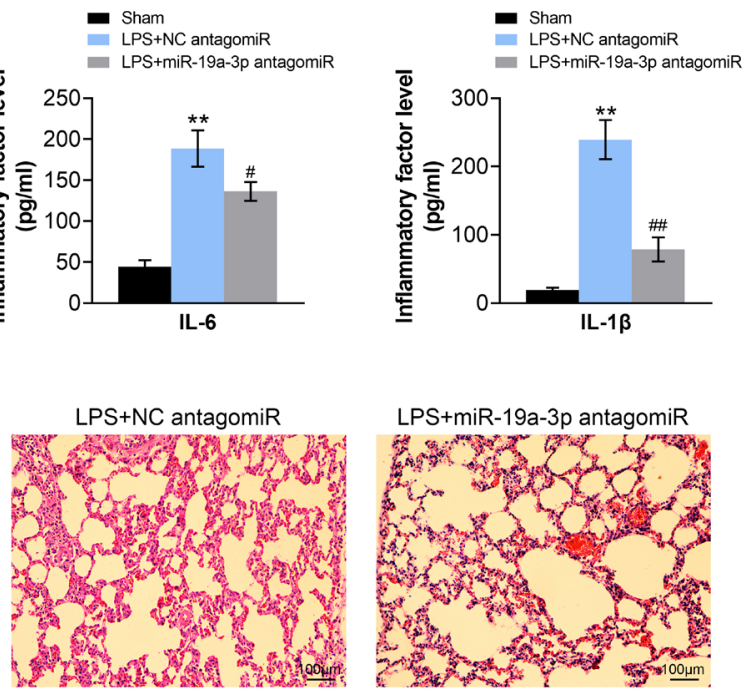

Figure 5. Knockdown of miR-19a-3p inhibited sepsis-induced lung damage in mice.

(A) The expression level of miR-19a-3p in mouse model was measured with qRT-PCR and Western blotting (B) The levels of TNF-a, IL-6 and IL-1 $\beta$ were measured using ELISA. (C) The lung damage in LPS treated mice was assessed with HE staining (Bar $=100 \mu m) .{ }^{*} p<0.01$, *denotes the comparison of LPS + NC antagomiR group and the sham group. \#p<0.05, \#p<0.01,"denotes the comparison of LPS + miR$19 a-3 p$ antagomiR group and LPS + NC antagomiR group.

pathways, which suggests that miRNAs can play a very important role in sepsis (Huang et al., 2014).

It has been shown that the miRNAs levels in serum differs between patients with sepsis and healthy individuals. For example, the expression level of miR-150s was significantly decreased in the serum of patients with sepsis, as measured with miRNA microarrays (Vasilescu et al., 2009). The expression level of miR-19a-3p was shown to be elevated in sepsis (Chen et al., 2019). In addition, it has also been reported that overexpression of miR-199a can exacerbate sepsis-induced acute lung injury (Liu et al., 2018). miR-145 could also negatively regulate sepsis-induced acute lung damage through TGFBR2 signaling pathway (Cao et al., 2019). LPS stimulation leads to inflammatory responses in human and mouse immune cells and contributes to the increase in several miRNAs expression (Liu et al., 2015). In this study we focused on miR-19a-3p role in sepsis. We employed LPS treatment to construct in vitro and in vivo sepsis models. In the MH-S in vitro cell model of lung injury we observed that the level of miR-19a-3p increased with LPS treatment and was correlated with the time of the treatment.. At the same time, miR-19a-3p expression was stable in untreated (control) MH-S cells, indicating that miR-19a-3p might be a potential marker of sepsis-induced lung injury.

The research from Huang and others revealed that the high expression of miR-19a is closely related to lymph node metastasis, and that miR-19a strongly affected $\mathrm{TNF}-\alpha-$ induced epithelial-mesenchymal transformation (EMT) in colorectal cancer cells (Huang et al., 2015). Some studies have shown that the 1 IL- 6 and TNF- $\alpha$ level in circulating blood were very low under normal circumstances, and can be significantly increased in inflammation (Ma et al., 2014). The levels of inflammatory factors such as IL- 6 and TNF- $\alpha$ reflected the severity of inflammatory damage and were related to the severity of disease (Wu \& Li, 2015). The results of the present study are consistent with the previous studies. We found that the levels of TNF- $\alpha$, IL- 6 and IL- $1 \beta$ were significantly increased in LPS-treated MH-S cells, andmiR-19a$3 \mathrm{p}$ inhibition alleviated this increase. Similarly, knockdown of miR-19a-3p in LPS-treated mice has also been found to alleviate the damage to lung tissues. Based on these results, we speculate that miR-19a-3p might be a pro-inflammatory molecular marker.

Ubiquitination plays an important role in TNF- $\alpha-$ mediated cell necrosis (Wu et al., 2014). USP13 can regulate the ubiquitination of an oncoprotein, enhance cellular immunity, and regulate DNA damage repair (Zhang et al., 2013). A recent study reported that down-regulation of USP13 may lead to an increase in LPS-induced lung injury (Li et al., 2019). At the same time, our target gene predictions showed that USP13 is one of the targets of miR-19a-3p, and we experimentally confirmed that miR19a-3p negatively regulated USP13 expression. Similar to previous studies, USP13 overexpression inhibited inflammatory factors' secretion. We show that the effect of miR-19a-3p knockdown on the secretion of inflammatory factors in LPS-treated MH-S cells can be reversed by USP13 knockdown.

In conclusion, miR-19a-3p knockdown could alleviate sepsis-induced lung injury by increasing USP13 expression, suggesting that miR-19a-3p may be an ideal marker and therapeutic target for the detection and treatment of sepsis-induced lung damage. Furthermore, due to the important role of miR-19a-3p in the inflammatory response, other target genes and signal pathways of miR-19a-3p should be studied in the future to provide new theoretical basis for the treatment of lung damage caused by sepsis.

\section{Acknowledgements}

Not applicable.

\section{Funding}

Not applicable. 


\section{Competing interests}

The authors state that there are no conflicts of interest to disclose.

\section{Ethics approval}

Ethical approval was obtained from the Ethics Committee of Nanjing First Hospital-Nanjing Medical University.

\section{Statement of Informed Consent}

Not applicable.

\section{Availability of data and materials}

All data generated or analyzed during this study are included in this published article.

\section{Authors' contributions}

Hangqi Ren designed the study and supervised the data collection, Wei $\mathrm{Mu}$ analyzed and interpreted the data, Qiaolian Xu prepared the manuscript for publication and reviewed the draft of the manuscript. All authors have read and approved the manuscript.

\section{REFERENCES}

Balthesen M, Messerle M, Reddehase MJ (1993) Lungs are a major organ site of cytomegalovirus latency and recurrence. J Virol 67: 5360-5366. https://doi.org/10.1128/JVI.67.9.5360-5366

Cao X, Zhang C, Zhang X, Chen Y, Zhang H (2019) MiR-145 negatively regulates TGFBR2 signaling responsible for sepsis-induced acute lung injury. Biomed Pharmacother 111: 852-858. https://doi. org/10.1016/j.biopha.2018.12.138

Chen J, Lin M, Zhang S (2019) Identification of key miRNA-mRNA pairs in septic mice by bioinformatics analysis. Mol Med Rep 20: 3858-3866. https://doi.org/10.3892/mmr.2019.10594

Dombrovskiy VY, Martin AA, Sunderram J, Paz HL (2007) Rapid increase in hospitalization and mortality rates for severe sepsis in the United States: a trend analysis from 1993 to 2003. Crit Care Med 35: 1244-1250. https://doi.org/10.1097/01.CCM.0000261890.41311.E9

Huang J, Sun ZD, Yan WY, Zhu YJ, Lin YX, Chen JJ, Shen BR, Wang J (2014) Identification of microRNA as sepsis biomarker based on miRNAs regulatory network analysis. Biomed Res Int 2014: 594350. https://doi.org/10.1155/2014/594350

Huang L, Wang X, Wen C, Yang X, Song M, Chen J, Wang C, Zhang B, Wang L, Iwamoto A, Wang J, Liu H (2015) Hsa-miR-19a is associated with lymph metastasis and mediates the TNF- $\alpha$ induced epithelial-to-mesenchymal transition in colorectal cancer. Sci Rep 5: 13350-13350. https://doi.org/10.1038/srep13350

Juroš GF, Nikić MT, Sarić SD, Perić M, Rogić D (2019) Contribution of presepsin, procalcitonin and C-reactive protein to the SOFA score in early sepsis diagnosis in emergency abdominal surgical patients. Signa Vitae: J Intesive Care Emerg Med 15: 38-45. https://doi. org/10.22514/SV151.042019.5

Li L, Wei J, Li S, Jacko AM, Weathington NM, Mallampalli RK, Zhao J, Zhao Y (2019) The deubiquitinase USP13 stabilizes the anti-inflammatory receptor IL-1R8/Sigirr to suppress lung inflam- mation. EBioMedicine 45: 553-562. https://doi.org/10.1016/j.ebiom.2019.06.011

Ling Y, Li ZZ, Zhang JF, Zheng XW, Lei ZQ, Chen RY, Feng JH. (2018) MicroRNA-494 inhibition alleviates acute lung injury through Nrf2 signaling pathway via NQO1 in sepsis-associated acute respiratory distress syndrome. Life Sci 210: 1-8. https://doi.org/10.1016/j. lfs.2018.08.037

Liu F, Li Y, Jiang R, Nie C, Zeng Z, Zhao N, Huang C, Shao Q, Ding C, Qing C, Xia L, Zeng E, Qian K (2015) miR-132 inhibits lipopolysaccharide-induced inflammation in alveolar macrophages by the cholinergic anti-inflammatory pathway. Exp Lung Res 41: 261-269. https://doi.org/10.3109/01902148.2015.1004206

Liu Y, Guan H, Zhang JL, Zheng Z, Wang HT, Tao K, Han SC, Su LL, Hu D (2018) Acute downregulation of miR-199a attenuates sepsis-induced acute lung injury by targeting SIRT1. Am J Physiol Cell Physiol 14: C449-C455. https://doi.org/10.1152/ajpcell.00173.2017

Livak KJ, Schmittgen TD (2001) Analysis of relative gene expression data using real-time quantitative PCR and the 2(-Delta Delta C(T)) Method. Methods 25: 402-408. https://doi.org/10.1006/ meth.2001.1262

Ma XJ, Guo CY, Yin HJ, Liu Y, Shi DZ (2014) Effect of activating blood circulation or activating blood circulation and detoxication on platelet activation, inflammation, and coagulation status in acute myocardial infarction rats. Zhongguo Zhong Xi Yi Jie He Za Zhi 34: 1329-1334 (in Chinese). PMID: 25566624

Hayakawa M (2018) Management of disseminated intravascular coagulation: current insights on antithrombin and thrombomodulin treatments. Open Access Emerg Med 10: 25-29. https://doi.org/10.2147/ OAEM.S135909

Opal SM (2014). The current understanding of sepsis and research priorities for the future. Virulence 5: 1-3. https://doi.org/10.4161/ viru. 26803

Sethi A, Kulkarni N, Sonar S, Lal G (2013) Role of miRNAs in CD4 $\mathrm{T}$ cell plasticity during inflammation and tolerance. Front Genet 4: 8. https://doi.org/10.3389/fgene.2013.00008 4: 8-.

Sotiropoulou G, Pampalakis G, Lianidou E, Mourelatos Z (2009) Emerging roles of microRNAs as molecular switches in the integrated circuit of the cancer cell. RNA 15: 1443-1461. https://doi. org/10.1261/rna.1534709

Stapleton RD, Wang BM, Hudson LD, Rubenfeld GD, Caldwell ES, Steinberg KP (2007) Causes and timing of death in patients with the acute respiratory distress syndrome. Chest 14: 58-61. https://doi. org $/ 10.1378 /$ chest.128.2.525

Wang JF, Yu ML, Yu G, Bian JJ, Deng XM, Wan XJ, Zhu KM (2010) Serum miR-146a and miR-223 as potential new biomarkers for sepsis. Biochem Biophys Res Commun 394: 184-188. https://doi. org/10.1016/j.bbrc.2010.02.145

Wu CJ, Conze DB, Li X, Ying SX, Hanover JA, Ashwell JD (2014) TNF- $\alpha$ induced c-IAP1/TRAF2 complex translocation to a Ubc6containing compartment and TRAF2 ubiquitination. EMBO J 24: 1886-1898. https://doi.org/10.1038/sj.emboj.7600649

Wu J, Li X (2015) Plasma tumor necrosis factor-alpha (TNF- $\alpha$ ) levels correlate with disease severity in spastic diplegia, triplegia, and quadriplegia in children with cerebral palsy. Med Sci Monit 21: 3868-3874. https://doi.org/10.12659/msm.895400

Vasilescu C, Rossi S, Shimizu M, Tudor S, Veronese A, Ferracin M, Nicoloso MS, Barbarotto E, Popa M, Stanciulea O, Fernandez MH, Tulbure D, Bueso-Ramos CE, Negrini M, Calin GA (2009) MicroRNA fingerprints identify miR-150 as a plasma prognostic marker in patients with sepsis. Plos One 4: e7405. https://doi.org/10.1371/ journal.pone.0007405

Zhang J, Zhang P, Wei Y, Piao HL, Wang W, Maddika S, Wang M, Chen D, Sun Y, Hung MC, Chen J, Ma L (2013) Deubiquitylation and stabilization of PTEN by USP13. Nat Cell Biol 15: 1486-1494. https://doi.org/10.1038/ncb2874 